\title{
A New Electron Microscope with an Easy Operation System for Nano Analysis
}

\author{
S. Kawai, M. Matsushita, K. Tanaka, T. Iwama, N. Endo, T. Kuba, Y. Ohkura, and Y. Kondo \\ JEOL, Ltd., 1-2 Musashino 3-chome, Akishima, Tokyo 196-8558, Japan.
}

Semiconductors and electronic devices are still rapidly moving towards a higher circuit integration and density as well as a narrower design rule. Quick and much feedbacks from the prototypes or the current products accelerate research and development of semiconductors and electronic devices. In order to evaluate the characters of those, it needs a great variety of information such as transmission electron microscope (TEM) images, scanning transmission electron microscope bright field (STEMBF) images, STEM-dark field (STEM-DF) images, secondary electron (SE) images, electron diffraction patterns (DPs), elemental maps with X-ray energy dispersive spectroscopy (EDS) and/or electron energy loss spectroscopy (EELS) at nano scale level. However, to obtain these kinds of informations, well-experienced operators after considerable effort are necessary. Therefore, a high throughput field emission electron microscope with ease of use is requested from these fields. JEM2800 has been developed to satisfy these requests: variety of information by inexperienced operators. This paper reports the characteristics of this new microscope for these requests.

1. Basic improvement of operations: TEM, STEM-BF, STEM-DF, SE images, and DPs can be observed by one-touch switch operation on a graphical user interface (GUI) as shown in Fig. 1. Automatic functions are added for inexperienced users. These are focus, stigmatism, Z-control, contrast \& brightness, and crystal orientation adjustment functions.

2. Hardwares to assist users: Field of view (FOV) and magnification are kept same, when one switches between the TEM mode and the scanning mode. Therefore, the TEM image and the scanning images (STEM-BF, STEM-DF and SE) can be observed very easy and speedily without wandering the FOV. Scanning images can be observed simultaneously to permits direct comparison of those images.

3. Advanced assist for users: When measuring critical dimension (CD) measurement of semiconductors, it is preferable to rotate the TEM image. The rotation function of CCD camera has been prepared in order to satisfy such requirement. Moreover, a newly designed operation panels simplify operations of those microscopy. Besides, the images and the pattern are recorded in a common digital image format (BITMAP and TIFF).

4. Navigation system: For inexperienced operators, the operation navigation system: "JEM-Navi", is prepared in JEM-2800 including a TEM and STEM glossary, which helps the users to learn the microscopy. Important operations can be learned and confirmed visually with the help of their animations stored in the JEM-Navi.

5. High resolution: High-resolution images can be observed: resolution of $0.1 \mathrm{~nm}$ for TEM lattice image and of $0.2 \mathrm{~nm}$ for STEM lattice image, and point resolution of $0.5 \mathrm{~nm}$ for SE image can be obtained. A STEM high-angle annular dark field (HAADF) image of a Si[110] is shown in Fig. 2. Spacing between two silicon columns $(0.136 \mathrm{~nm})$ is clearly resolved. 
The performances of JEM-2800 is summarized in Table 1. We believe that this microscope is accepted in a wide field of manufacturing as well as of research and development as an indispensable instrument for nano analysis.

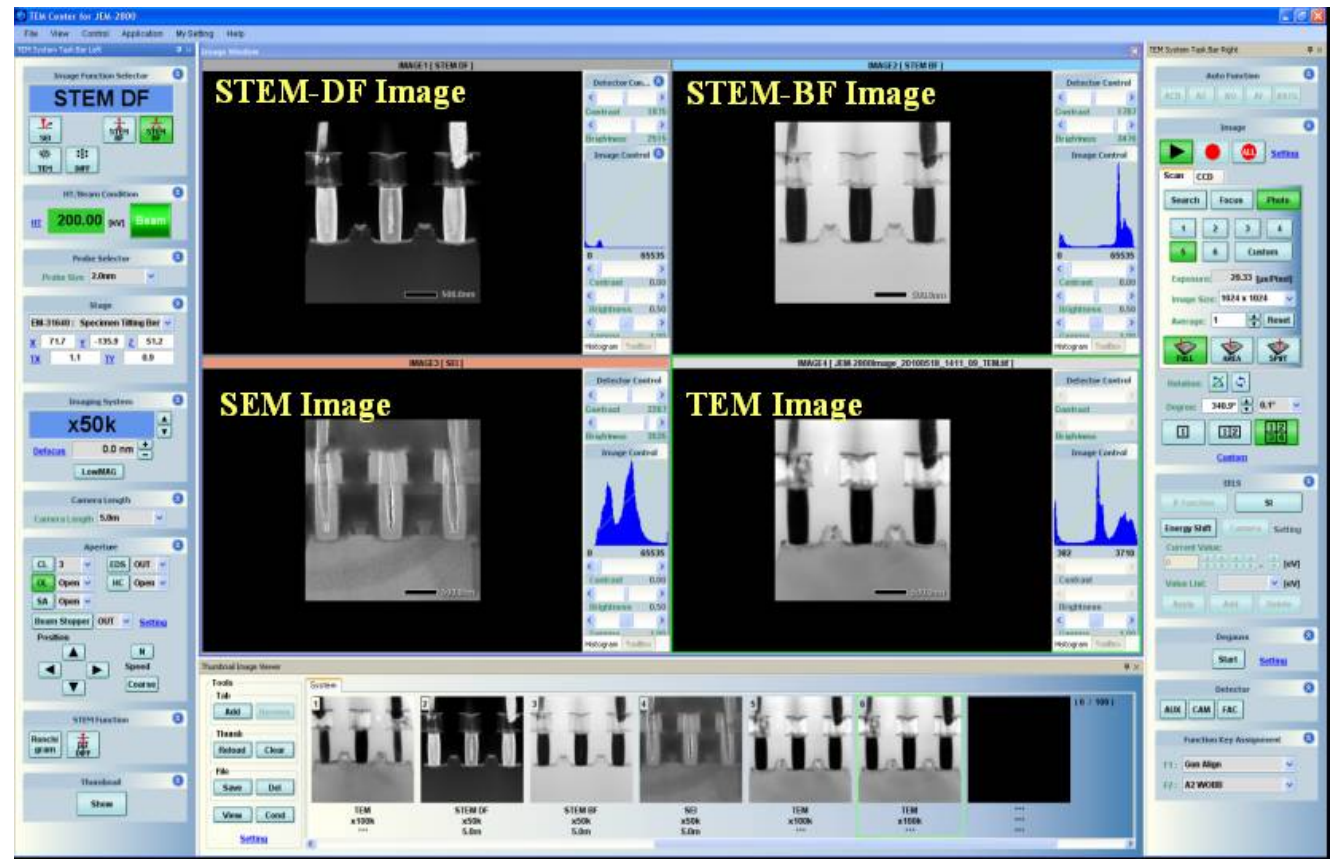

Fig. 1 Graphical user interface

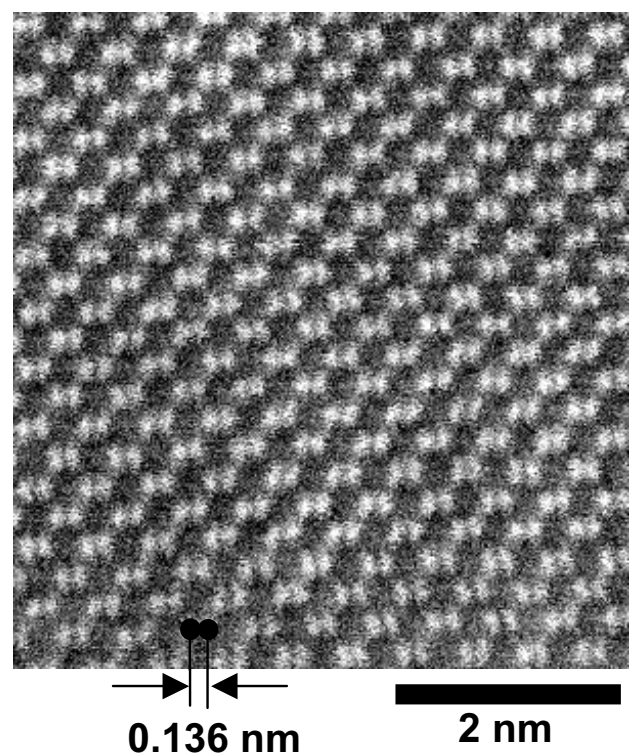

Fig. 2 A STEM-HAADF image of a $\mathrm{Si}[110]$ single crystal taken at $200 \mathrm{kV}$.
Table1. Performances and charactors of JEM-2800

\begin{tabular}{|c|c|}
\hline Acc. Voltage & $200 \mathrm{kV}, 100 \mathrm{kV}$ \\
\hline Electron Gun & $\mathrm{ZrO} / \mathrm{W}(100)$ Schottky \\
\hline \multirow{5}{*}{$\begin{array}{c}\text { Resolution } \\
(200 \mathrm{kV})\end{array}$} & TEM: $\quad 0.1 \mathrm{~nm}$ (lattice) \\
\hline & $0.21 \mathrm{~nm}$ (point) \\
\hline & $0.2 \mathrm{~nm}$ (lattice) \\
\hline & (0.136 $\mathrm{nm}$ attainable) \\
\hline & $0.5 \mathrm{~nm}$ (point) \\
\hline \multirow[t]{4}{*}{ Image } & TEM \\
\hline & Diffraction \\
\hline & STEM (BF, DF) \\
\hline & $\frac{\text { SE Image }}{\mathrm{Y} \cdot+25^{\circ} \mathrm{Y} \cdot+30^{\circ}}$ \\
\hline Tilting Angle & $\mathrm{X}: \pm 25^{\circ}, \mathrm{Y}: \pm 30^{\circ}$ \\
\hline Spec. Movement & $\pm 1 \mathrm{~mm}(\mathrm{X}, \mathrm{Y}), \pm 0.2 \mathrm{~mm}(\mathrm{Z})$ \\
\hline TEM Mag. & $\times 500-\times 20,000,000$ \\
\hline STEM Mag. & $\times 100-\times 150,000,000$ \\
\hline SE Image Mag. & $\times 100-\times 150,000,000$ \\
\hline Camera Length & $0.6-25 \mathrm{~m}$ \\
\hline (on 24-inch Wide LCD) & \\
\hline
\end{tabular}

